\title{
Generation of optical phase singularities by computer-generated holograms
}

\author{
N. R. Heckenberg, R. McDuff, C. P. Smith, and A. G. White \\ Department of Physics, University of Queensland, Saint Lucia, Brisbane, Australia 4072
}

Received September 23, 1991

Laser beams that contain phase singularities can be generated with computer-generated holograms, which in the simplest case have the form of spiral Fresnel zone plates.

There has recently been considerable interest in optical fields that exhibit phase singularities, which manifest themselves as isolated dark spots in the modal patterns of certain lasers. Each dark spot has a topological charge that represents the number of $2 \pi$ accumulated when the phase gradient is integrated around it. The wave fronts near a singularity have a helical structure, while the field at the singularity must be zero because of the ambiguous phase; hence the dark spot.

It has been shown theoretically how, under the influence of nonlinear interactions, frequencydegenerate transverse modes can lock together with a fixed phase difference to produce stable patterns that contain one or more of the singularities. ${ }^{1}$ Such modal patterns have also been observed experimentally. ${ }^{2-4}$ Uncontrolled generation of random arrays of singularities has also been reported. ${ }^{5}$

Here we report a means of generating such singularities in a controlled way, using only the simplest equipment, which amounts to the use of a computergenerated hologram, or zone plate. The hologram that is simulated is that of a modal pattern that contains a set of phase singularities using a reference plane wave. The technique can be extended to produce patterns of great complexity, but in this Letter we mainly concentrate on the circularly symmetric doughnut mode.

If one interferes two coherent optical fields $E_{1} \exp \left(i \phi_{1}\right)$ and $E_{2} \exp \left(i \phi_{2}\right)$, the resultant spatial intensity pattern is modulated by a $2 E_{1} E_{2} \cos \left(\phi_{1}+\phi_{2}\right)$ term, which represents the interference fringes. The desired interference pattern is between a plane wave and the lowest-order hybrid doughnut mode, ${ }^{3}$ $E_{0} r / \omega \exp \left[-(r / w)^{2}\right] \exp \left(-i k r^{2} / 2 R \pm i \theta\right)$. Here $k$ is the wave number, $\omega$ is the spot size, and $R$ is the radius of curvature of the doughnut Gaussian beam. The plus or minus refers to the two possible chiralities of the helical wave. The boundary between a light fringe and a dark fringe occurs when the above cosine term is zero, and when the beams are collinear, this means

$$
\pm \theta=(n+1 / 2) \pi+\frac{k r^{2}}{2 R}
$$

$(n=0, \pm 1, \pm 2, \ldots)$, which is the equation of a double spiral. We can now produce a binary hologram (or

zone plate) by filling in the region where the modulation term is negative. This produces the pattern shown in Fig. 1(a), which may be recognized as a variation on the Fresnel zone plate. Like a normal zone plate, it will tend to form foci at distances such that the waves from all over the transparent areas will arrive in phase, but unlike normal zone plates, symmetry is broken on the axis as waves from diametrically opposite regions will always cancel out. When such a hologram is illuminated with coherent light, a helical wave will be reconstructed. Equation (1) can be generalized for charge $p$ doughnut modes; i.e.,

$$
p \theta=(n+1 / 2) \pi+\frac{k r^{2}}{2 R}
$$

$(n=0, \pm 1, \pm 2, \ldots)$. A charge 2 zone plate is shown in Fig. 1(b). Note that $p=0$ corresponds to the standard Fresnel zone plate.

If the two interfering beams are not collinear (i.e., misdirected) then an extra term is added onto Eq. (2), which becomes

$$
p \theta=(n+1 / 2) \pi+\frac{k r^{2}}{2 R}+k r \sin \gamma \cos \theta
$$

$(n=0, \pm 1, \pm 2, \ldots)$, where $\gamma$ is the angle of misdirection. If the radius of curvature $R$ is large, then a characteristic pattern as shown in Fig. 2 is produced. The clear signature of the presence of a phase singularity is a defect in the fringe pattern where a new fringe starts at the location of the singularity. Not only is the pattern of Fig. 2 the basis of an off-axis

(a)

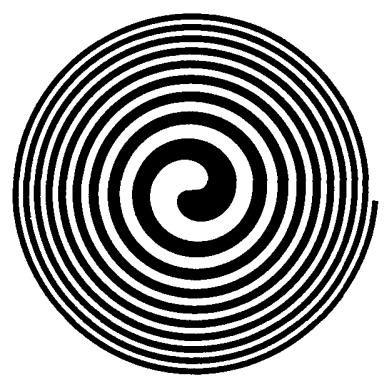

Fig. 1. Computer-generated zone plates. (a) Charge 1. (b) Charge 2. (b)

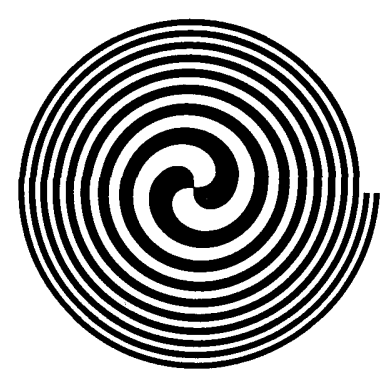




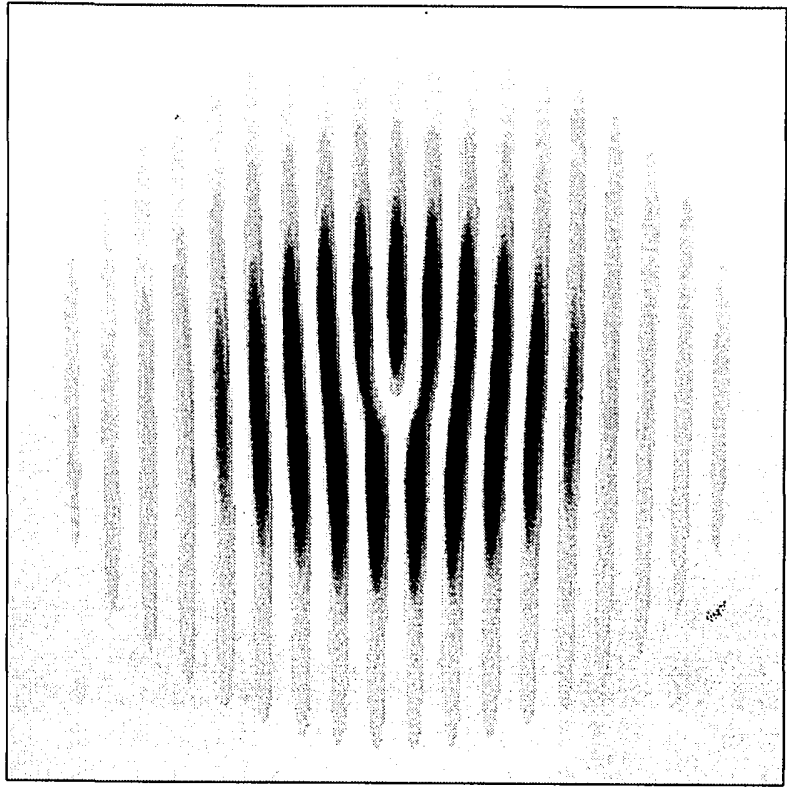

Fig. 2. Theoretical interference pattern produced by interfering a Gaussian Hermite $\mathrm{TEM}_{01}{ }^{*}$ hybrid mode containing a singly charged singularity with a slightly inclined plane wave. The signature of a phase singularity is the defect in the fringe structure where an interference fringe starts at the location of the singularity.

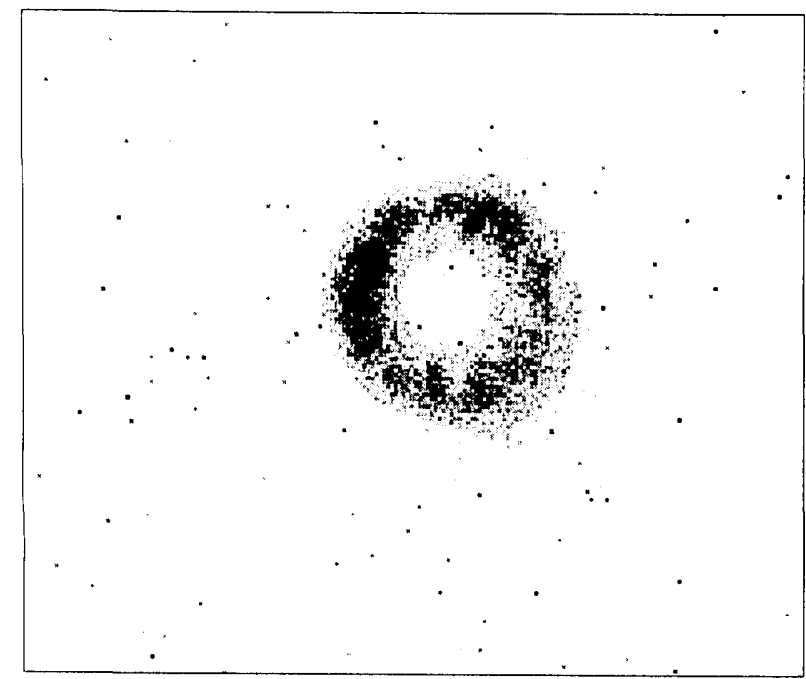

Fig. 3. CCD camera photograph of a typical reconstruction of a Gaussian Hermite TEM $_{01}{ }^{*}$ hybrid mode (or doughnut mode) by $\mathrm{He}-\mathrm{Ne}$ laser illumination of the zone plate represented by Fig. 1(a).

hologram for helical wave production, but it is also the key to the subsequent detection of such waves by interferometry. ${ }^{4}$

A number of spiral zone plates were made by photoreducing laser-printed patterns of A4 size $(210 \mathrm{~mm} \times 297 \mathrm{~mm}$ ) generated by a microcomputer. An appropriate scale is determined through Eq. (2), which indicates that when a plate is illuminated by a plane wave, a focus will be produced at a distance $R$, which is conveniently made to be approximately $30 \mathrm{~cm}$. Plates for both charge 1 and $2(2 \pi$ and $4 \pi$ phase integrals) doughnuts were made; the helicity can be changed by reversing the plate. Normal (circular) zone plates were also made in the same way for comparison. All the plates were able to form images of bright objects such as the Sun. Under illumination from a spatially filtered $\mathrm{He}-\mathrm{Ne}$ laser, the zone plates, including the standard Fresnel zone plates, produced the expected family of focal regions. To separate the fundamental beam from the undiffracted light and higher-order Fresnel images, a 5 -cm focal-length lens was placed at the fundamental focus. The lens passed the helical wave but caused the unwanted light to focus and then spread rapidly. The helical doughnut mode produced by the spiral zone plate was then easily viewed on a screen several meters away and was recorded by an EDC-1000 CCD camera with a resolution of 196 pixels by 165 pixels and 256 levels of gray. Note that in the examples shown, bright areas appear black.

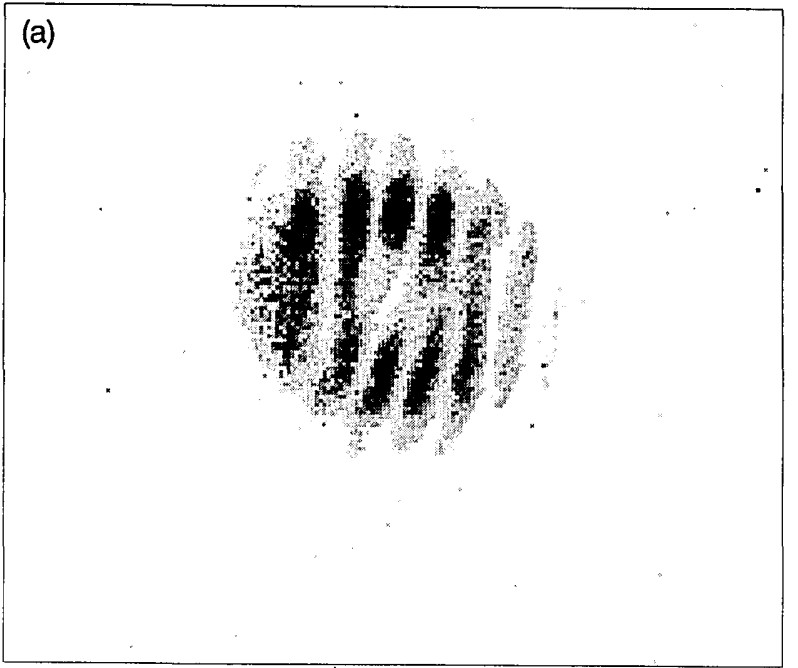

(b)

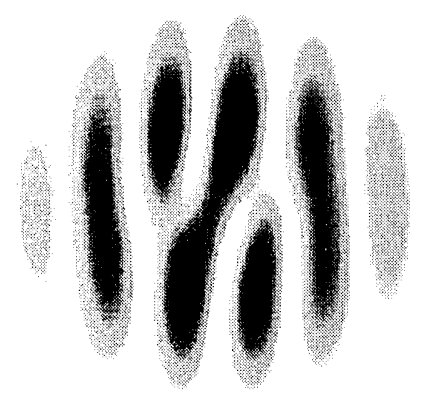

Fig. 4. (a) CCD picture of interference fringes obtained by interfering two doughnut beams in the Mach-Zehnder interferometer setup. By comparison with Fig. 2, we see directly the occurrence of the phase singularities of each beam. (b) Theoretical interference pattern produced by interfering two misaligned and misdirected doughnut modes. Again we see the two signatures of the phase singularities and the similarity to (a). 


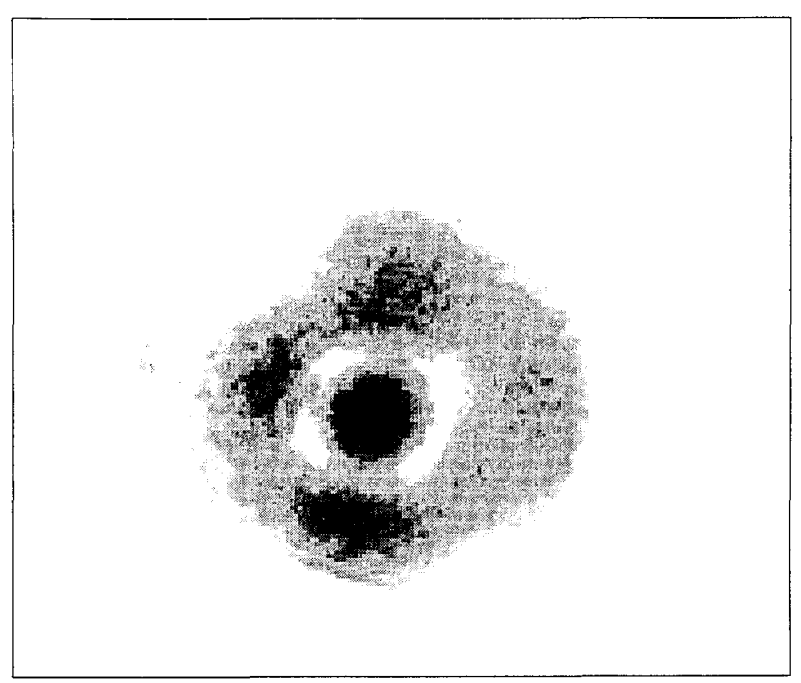

Fig. 5. Optical leopard produced by a hologram designed to reconstruct a Gaussian Hermite TEM $_{20}{ }^{*}$ hybrid mode.

A typical helical doughnut mode produced by the charge 1 plate is shown in Fig. 3. The fact that the central minimum is persistent, not being filled in by diffraction over many meters of propagation, is an indication that a phase singularity is present, as can be confirmed by interferometry. A convenient way to do this is to use a Mach-Zehnder interferometer to duplicate the irradiance pattern and to interfere the two slightly displaced beams together. ${ }^{4-6}$ The result is two positions where a singularity is superimposed on a region of relatively constant phase similar to the local region around the singularity in Fig. 2. The extra fringe generated at each singularity is clearly visible in Fig. 4(a). Figure 4(b) shows for comparison the theoretical irradiance distribution calculated by using the full equations for two charge 1 doughnuts that are slightly misaligned and misdirected. Similarly, two extra fringes appear in the charge 2 case.

Although we have shown clearly that spiral zone plates can generate helical modes, they suffer many problems common to on-axis holograms of the Gabor type, particularly the lack of separation of the reconstructed beams from each other and the incident light, as well as the multiplicity of images generated by the binary nature of the pattern. Several sinusoidal holograms of various charges were made by assigning a linear gray scale only to the cosine interference term mentioned above at the highest spatial resolution achievable for our particular laser printer. These holograms produced helical beams similar in quality to those of the zone plates in the same apparatus without the problem of multiple orders. Since these results are similar to those of the zone plates, they will not be shown here. However, shown in Fig. 5 is the beam produced by a hologram designed to produce a Gaussian Hermite $\mathrm{TEM}_{20}{ }^{*}$ hybrid mode, or optical leopard. ${ }^{2}$ This beam consists of a pair of Gaussian Hermite TEM $_{20}$ and TEM ${ }_{02}$ modes of equal amplitude locked together with a $\pi / 2$ phase shift. This combination produces a pattern of five bright peaks and four singly charged singularities, two positive and two negative, arranged on the vertices of a square, with diametrically opposed singularities having the same charge. For complex sets of singularities, holograms are computationally easier to produce than their corresponding zone plates since no boundaries need be found.

Our results show that it is relatively easy to produce optical fields that contain simple patterns of phase singularities, previously obtained only through nonlinear interactions in lasers, by simple optical techniques that involve computer-generated holograms, or zone plates. Not only does this greatly simplify the study of possible applications of such beams, but the holograms can be used in further optical techniques such as Fourier optic pattern recognition to identify the presence of singularities in complex fields.

This research was supported by the Australian Research Council.

\section{References}

1. L. A. Lugiato, C. Oldano, and L. M. Narducci, J. Opt. Soc. Am. B 5, 879 (1989).

2. M. Brambilla, F. Battipede, L. A. Lugiato, V. Penna, F. Prati, Chr. Tamm, and C. O. Weiss, Phys. Rev. A 43, 5090 (1991).

3. Chr. Tamm and C. O. Weiss, J. Opt. Soc. Am. B 7, 1034 (1990).

4. A. G. White, C. P. Smith, N. R. Heckenberg, H. Rubinsztein-Dunlop, R. McDuff, C. O. Weiss, and Chr. Tamm, J. Mod. Opt. 38, 2531 (1991).

5. N. B. Baranova, B. Ya. Zel'dovich, A. V. Mamaev, N. F. Pilipetskil, and V. V. Shkukov, Sov. Phys. JETP 33, 195 (1981).

6. J. M. Vaughan and D. V. Willets, J. Opt. Soc. Am. 73, 1018 (1983). 